OPEN ACCESS

Edited by:

Tzvi Dwolatzky,

Technion Israel Institute of

Technology, Israel

Reviewed by:

Pier Giorgio Cojutti,

University of Udine, Italy

Hideshi Okada,

Gifu University, Japan

*Correspondence:

Hongxia Li

Ihxs/s@sina.com

Guogang Xu

guogang_xu@163.com

tThese authors have contributed equally to this work

Specialty section: This article was submitted to

Geriatric Medicine,

a section of the journal

Frontiers in Medicine

Received: 10 September 2020 Accepted: 18 January 2021

Published: 25 February 2021

Citation:

Liu T, Hu C, Wu J, LiU M, Que Y, Wang J, Fang $X, X u G$ and Li H (2021)

Incidence and Associated Risk

Factors for Lactic Acidosis Induced by

Linezolid Therapy in a Case-Control

Study in Patients Older Than 85 Years.

Front. Med. 8:604680.

doi: 10.3389/fmed.2021.604680

\section{Incidence and Associated Risk Factors for Lactic Acidosis Induced by Linezolid Therapy in a Case-Control Study in Patients Older Than 85 Years}

\author{
Tingting $\mathrm{Liu}^{1 \dagger}$, Chao $\mathrm{Hu}^{2 \dagger}$, Jionghe $\mathrm{Wu}^{1+}$, Miao $\mathrm{Liu}^{3}$, Yifan Que ${ }^{2}$, Jiang Wang ${ }^{4}$, \\ Xiangqun Fang ${ }^{1}$, Guogang $\mathrm{Xu}^{2 *}$ and Hongxia $\mathrm{Li}^{1 *}$
}

${ }^{1}$ Department of Pulmonary and Critical Care Medicine, The Second Medical Center, National Clinical Research Center for Geriatric Diseases, Chinese People's Liberation Army General Hospital, Beijing, China, ${ }^{2}$ The Second Medical Center, National Clinical Research Center for Geriatric Diseases, Chinese People's Liberation Army General Hospital, Beijing, China,

${ }^{3}$ Second Medical Centre, Institute of Gerontology, Chinese People's Liberation Army General Hospital, Beijing, China,

${ }^{4}$ Centre of Pulmonary and Critical Care Medicine, Chinese People's Liberation Army General Hospital, Beijing, China

Background: Serum lactic acid is considered a prognostic indicator in critically ill patients. However, studies on linezolid-induced lactic acidosis (LILA) are still limited. Individuals older than 85 years old (very elderly) have limited capacity for organ compensation, and LILA data from these patients are lacking. In this study, we evaluated the risk factors for LILA in patients older than 85 years and established a risk prediction model for geriatric practice.

Methods: In this retrospective cohort study, blood gas analysis data and arterial lactate levels were monitored in patients older than 85 years during the use of teicoplanin or linezolid. After propensity score matching analyses, we compared the incidence of lactic acidosis between the teicoplanin and linezolid therapy groups and identified the risk factors of LILA.

Results: The incidence of lactic acidosis was found to be much lower in the group receiving teicoplanin than those receiving linezolid therapy (0 vs. 35.7\%; $p<0.0001$ ). A duration of linezolid therapy $\geq 9$ days [odds ratio (OR), 3.541; 95\% confidence interval (Cl), 1.161-10.793; $p=0.026$ ], an arterial blood glucose level $\geq 8 \mathrm{mmol} / \mathrm{L}(\mathrm{OR}, 4.548$; $95 \% \mathrm{Cl}, 1.507-13.725 ; p=0.007)$, and a high sequential organ failure assessment score (OR, 1.429; 95\% Cl, 1.213-1.685; $p<0.0001)$ were risk factors for LILA. The constructed risk model could be used to predict LILA (area under the curve, 0.849; specificity, $65.1 \%$; sensitivity, $91.4 \%$, with a negative predictive value of $93.2 \%$ and a positive predictive value of $59.3 \%)$.

Conclusions: LILA can occur in patients older than 85 years after a relatively shorter duration of linezolid therapy. Therefore, close monitoring of blood gas and arterial lactate levels during linezolid therapy in the very elderly population is necessary.

Keywords: risk prediction model, risk factors, linezolid-induced lactic acidosis, very elderly, serum lactic acid 


\section{INTRODUCTION}

Serum lactic acid is produced by anaerobic glycolysis, mainly in the skeletal muscles, skin, erythrocytes, and central nervous system (1). Clinically, elevated lactate levels often represent hypoxia in tissues, so lactate is commonly used to evaluate tissue perfusion and prognosis in critically ill patients (2, 3). It has been reported that when the blood lactate level is higher than $10 \mathrm{mmol} / \mathrm{L}$, the mortality rate is $>80 \%$ $(4,5)$.

Elevated lactate levels caused by drugs do not necessarily indicate hypoxia, and such high lactate levels gradually decrease back to the normal range after drug withdrawal. Among different types of drug-induced lactic acidosis, little is known about linezolid-induced lactic acidosis (LILA). Linezolid is the first clinically available oxazolidinone antibacterial agent against infections caused by multidrugresistant gram-positive pathogens (6-8). The most common adverse reaction to linezolid is reversible myelosuppression (anemia, thrombocytopenia, and leukopenia) (9), and some rare adverse reactions are toxic optic neuropathy $(10,11)$, irreversible peripheral neuropathy $(12-14)$, and lactic acidosis $(15,16)$. The incidence of LILA has been reported to be between 5 and $33 \%$, affecting the survival of patients (17-20).

However, large-sample studies on the risk factors for LILA and relevant data on the very elderly population are less reported so far. Hence, we analyzed the risk factors for LILA and established a risk prediction model.

\section{METHODS}

\section{Study Design and Participants}

This retrospective cohort case-control study was conducted at the Second Medical Centre of Chinese People's Liberation Army General Hospital.

Blood gas analysis and arterial lactate levels of patients older than 85 years were monitored during teicoplanin or linezolid treatment from October 2016 to April 2019 in our hospital. To compare lactic acidosis incidence between patients receiving teicoplanin and linezolid therapy, the baseline characteristics of patients in those groups were adjusted using propensity score matching. Patients receiving linezolid therapy were divided into the lactic acidosis and non-lactic acidosis groups, and the risk factors for LILA were evaluated.

Patients with shock, patients with respiratory failure (partial pressure of oxygen $\leq 60 \mathrm{mmHg}$ ) or liver failure (Child-Pugh classification C), patients with heart failure (NYHA classification 3 or higher), and those using drugs that affect lactate levels (such as metformin, salicylates, and nucleotide reverse transcriptase inhibitors) or receiving renal replacement therapy were excluded.

Patients older than 85 years had a low compensatory ability, with lactic acidosis defined as a serum $\mathrm{pH}<7.35$ and arterial lactate level $\geq 3.5 \mathrm{mmol} / \mathrm{L}$ in the study. End-point referred to the time when the medication was stopped. The baseline and end-point lactate levels were obtained from blood gas analyses performed when teicoplanin or linezolid therapy was started and stopped. An arterial blood collection syringe (BD Preset, UK) was used to collect $1 \mathrm{ml}$ of radial artery blood, which will be sent for testing within $15 \mathrm{~min}$. A blood gas analyzer (Roche Cobas B221, Switzerland) was used to analyze arterial blood $\mathrm{pH}$ and lactate levels. The World Health OrganizationThe Uppsala Monitoring Centre Method was used to evaluate LILA, and the causal relationship was certain or probable/likely to be classified as LILA. Linezolid was from Pfizer (USA) with two different formulations: linezolid injection $(0.6 \mathrm{~g}: 300 \mathrm{ml})$ and linezolid tablet $(0.6 \mathrm{~g})$. The dosage regimen was administrated as linezolid injection $(0.6 \mathrm{~g} \mathrm{qm})$ and linezolid tablet $(0.6 \mathrm{~g} \mathrm{qn})$ for all patients.

\section{Data Collection}

Baselines of the following clinical and laboratory variables were collected retrospectively from the electronic medical records system: sex, age, duration of linezolid therapy, infection site, the use of invasive ventilation, comorbid diseases such as chronic obstructive pulmonary disease, pulmonary fibrosis, coronary heart disease, hypertension, atrial fibrillation, diabetes mellitus), chronic kidney disease (CKD), neurological disease, malignant tumor, and thyroid hypofunction; laboratory indexes including the levels of serum creatinine, albumin, hemoglobin, creatine kinase, lactate dehydrogenase, alanine dehydrogenase, aspartate transaminase, troponin I (TNI), pro-brain natriuretic peptide, D-dimer, arterial blood glucose and estimated glomerular filtration rate (eGFR), sequential organ failure assessment (SOFA) score, and arterial lactate levels at baseline and the endpoint. SOFA score was assessed when starting teicoplanin or linezolid treatment.

\section{Statistical Analysis}

Quantitative data with normal distributions were expressed in the form of means and standard deviations and were analyzed by $t$-tests. Data with non-normal distributions were conveyed as medians and interquartile ranges and were assessed with the Mann-Whitney U test. Moreover, categorical variables were described as frequencies, with comparisons being made by using the chi-square test. To adjust for the significant differences in patients' baseline characteristics in the two treatment groups, we performed propensity score matching by implementing the nearest-neighbor matching in a 1:1 ratio. Factors with significant differences in univariate analysis were entered into a multivariate binary logistic regression model (forward: LR) to determine their independent effects. The results of the binary logistic regression model are presented as odds ratios (ORs) and the associated 95\% confidence intervals (CIs). The sensitivity and specificity of the risk prediction model were tested using the receiver operating characteristic curve analysis. The 30 -day mortality rates of patients in the lactic acidosis and non-lactic acidosis groups were assessed by the Kaplan-Meier method with the Breslow test. Variables with $p$-values $<0.05$ were considered 


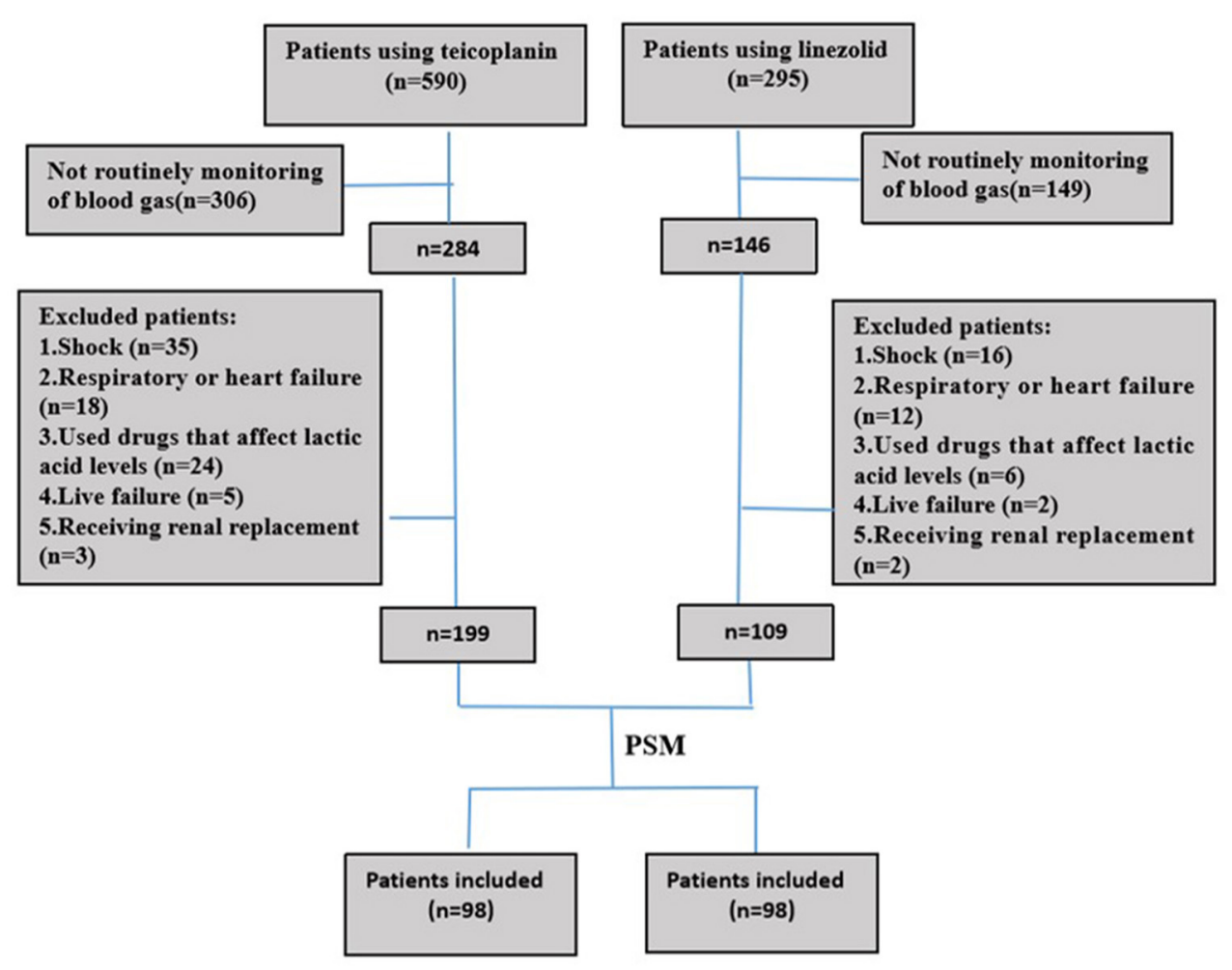

FIGURE 1 | Screening of patients using teicoplanin or linezolid during the study period.

statistically significant. All analyses were performed using IBM SPSS statistical software package version 23.0 (SPSS, Chicago, IL, USA).

\section{RESULTS}

\section{Patient Characteristics and Clinical Factors}

In this retrospective cohort study, 199 and 108 patients were administered teicoplanin or linezolid therapy, respectively (Figure 1). Of the patients, 15.3\% (15/98) were skin and soft tissue infections, and most were single streptococcal infections. Pulmonary infections were found in $84.7 \%(83 / 98)$ of the patients, mainly hospital-acquired pneumonia or ventilator-associated pneumonia. They were mixed infections based on gram-negative bacteria (Acinetobacter baumannii, Klebsiella pneumoniae, or Pseudomonas aeruginosa). All patients received the standard dose of antibiotics. Patient characteristics and clinical factors such as infection site, underlying disease (coronary heart disease, atrial fibrillation, and neurological disease), and SOFA scores are shown in Supplementary Material. Propensity score matching was used to adjust for significant differences in the baseline characteristics of the two groups. As a result, 98 patients were matched in each of the teicoplanin and linezolid therapy groups (Figure 1). The balance in baseline characteristics between the two groups improved considerably (Table $\mathbf{1}$ ).

\section{Arterial Lactate Levels at Baseline and the End-Point in Patients in the Teicoplanin and Linezolid Therapy Groups}

After propensity score matching, the incidence rates of lactic acidosis in the teicoplanin and linezolid therapy groups were $0 \%$ (0/98) vs. $35.7 \%$ (35/98), respectively, with significant differences $(p<0.0001$, Table 2). No significant difference was found in the baseline of arterial lactate levels between the two groups. In contrast, the arterial lactate level at the end-point was significantly higher in the linezolid therapy group than that in the teicoplanin therapy group ( $p<0.0001$, Table 2$)$.

\section{Univariate Analysis of Risk Factors for Linezolid-Induced Lactic Acidosis}

In the linezolid therapy group, there were 35 patients with lactic acidosis and 63 patients with non-lactic acidosis. The median duration of linezolid therapy was $10(7,12)$ days in the lactic acidosis group and $8(5,11)$ days in the non-lactic acidosis group $(p=0.053$, Table 3$)$. The numbers of patients with CKD in the lactic acidosis and non-lactic acidosis groups were 24 (68.6\%) and $31(49.2 \%)(p=0.064$, Table 3), respectively. Arterial lactate levels at the end-point were significantly different between the two groups $[4.6(3.7,5.5) \mathrm{mmol} / \mathrm{L}$ vs. $2.1(1.6,2.6) \mathrm{mmol} / \mathrm{L}$, $p<0.0001$, Table 3]. The clinical parameters, such as serum creatinine, hemoglobin, TNI, D-dimer, arterial blood glucose levels, and eGFR were significantly different between the two groups $(p<0.05$, Table 3 ). The SOFA scores of the two groups 
TABLE 1 | Patient demographic and clinical characteristics after propensity score matching (nearest neighbor matching).

\begin{tabular}{|c|c|c|c|}
\hline & Teicoplanin $(n=98)$ & Linezolid $(n=98)$ & $P$-value \\
\hline Male sex, $N(\%)$ & 88 (89.8\%) & 89 (90.8\%) & $0.809^{\star}$ \\
\hline Age, years, median (IQR) & $94(91,96.25)$ & $94(91,97)$ & $0.739^{\#}$ \\
\hline Duration of linezolid, days, median (IQR) & $8.5(6.75,12.00)$ & $9(6,11.25)$ & $0.937^{\#}$ \\
\hline Infection sites, $N(\%)$ & & & $0.400^{\star}$ \\
\hline Pulmonary infection & 87 (88.8\%) & 83 (84.7\%) & \\
\hline Non-pulmonary infection & $11(11.2 \%)$ & $15(15.3 \%)$ & \\
\hline Invasive ventilation, $N(\%)$ & $38(38.8 \%)$ & $34(34.7 \%)$ & $0.553^{\star}$ \\
\hline \multicolumn{4}{|l|}{ Underlying disease } \\
\hline COPD, N (\%) & $80(81.6 \%)$ & 78 (79.6\%) & $0.718^{\star}$ \\
\hline Pulmonary fibrosis, $N(\%)$ & $13(13.3 \%)$ & $11(11.2 \%)$ & $0.663^{\star}$ \\
\hline Coronary heart disease, $N(\%)$ & & & $0.817^{\star}$ \\
\hline Stable & 88 (89.8\%) & 87 (88.8\%) & \\
\hline Coronary ischemia & $10(10.2 \%)$ & $11(11.2 \%)$ & \\
\hline Hypertension, N (\%) & 79 (80.6\%) & 78 (79.6\%) & $0.858^{\star}$ \\
\hline Atrial fibrillation, $N(\%)$ & $53(54.1 \%)$ & 44 (44.9\%) & $0.199^{*}$ \\
\hline Diabetes mellitus, $N(\%)$ & 45 (45.9\%) & 43 (43.9\%) & $0.774^{*}$ \\
\hline Chronic kidney disease, $N(\%)$ & $46(46.9 \%)$ & 49 (50.0\%) & $0.668^{\star}$ \\
\hline Neurological disease, $N(\%)$ & $30(30.6 \%)$ & $29(29.6 \%)$ & $0.876^{\star}$ \\
\hline Malignant tumor, $N(\%)$ & $11(11.2 \%)$ & $12(12.2 \%)$ & $0.824^{*}$ \\
\hline Thyroid hypofunction, N (\%) & $3(3.1 \%)$ & $5(5.1 \%)$ & $0.718^{*}$ \\
\hline Serum creatinine, mg/dl, median (IQR) & $124.0(78.0,160.0)$ & $110.5(72.75,160.0)$ & $0.257^{\#}$ \\
\hline SOFA, median (IQR) & $9(6,13)$ & $9(6,12)$ & $0.233^{\#}$ \\
\hline Lactic acidosis, $N(\%)$ & 0 & 35 (35.7\%) & $<0.0001^{*}$ \\
\hline
\end{tabular}

IQR, interquartile range; COPD, chronic obstructive pulmonary disease; SOFA, sequential organ failure assessment.

${ }^{\star}$ Chi-square test.

\#Mann-Whitney $U$ test.

TABLE 2 | Arterial lactate at baseline and the end-point of patients.

Teicoplanin $(n=98)$ Linezolid $(n=98) \quad P$-value

\section{Arterial lactate, (mmol/L)}

$\begin{array}{lccc}\text { At baseline } & 1.2(0.9,1.525) & 1.2(0.9,1.4) & 0.450^{\#} \\ \text { At end-point } & 1.1(0.9,1.425) & 2.6(1.875,3.7) & <0.0001^{\#} \\ P \text { value } & 0.312^{\#} & <0.0001^{\#} & \\ \text { Lactic acidosis, N (\%) } & 0 & 35(35.7 \%) & <0.0001^{\#}\end{array}$

\#Mann-Whitney $U$ test.

were $10(9,15)$ and $6(5,9)(p<0.0001$, Table 3$)$, respectively. The 30 -day mortality rates were 48.6 and $28.6 \%$, respectively, and were significantly different ( $p=0.015$, Table 3, Figure 2).

\section{Risk Factors Associated With Linezolid-Induced Lactic Acidosis According to Multivariate Binary Logistic Regression}

Significant factors $(p<0.1)$ in the univariate analysis were entered into a multivariate binary logistic regression model to determine their independent effects. No associations were observed between LILA and CKD, serum creatinine levels, hemoglobin levels, TNI levels, pro-brain natriuretic peptide levels, D-dimer levels, or eGFR. Duration of linezolid therapy $\geq$
9 days (OR, 3.541; 95\% CI, 1.161-10.793; $p=0.026$, Table 4), arterial blood glucose level $\geq 8 \mathrm{mmol} / \mathrm{L}$ (OR, 4.548; 95\% CI, 1.507-13.725; $p=0.007$, Table 4), and a high SOFA score (OR, 1.429; 95\% CI, 1.213-1.685; $p<0.0001$, Table 4) were associated with LILA.

\section{Establishment of the Risk Prediction Model}

The risk of LILA can be predicted by three factors: the duration of linezolid therapy, arterial blood glucose level, and SOFA score.

Logit $(P)=-5.263+1.264 \times$ duration of linezolid $(\geq 9=1$, $<9=0)+1.515 \times$ arterialblood glucose $(\geq 8=1,<8=0)+$ $0.357 \times$ SOFA score.

The probability of LILA in each patient: $P=\mathrm{e}^{\operatorname{Logit}(P)} /(1+$ $\left.\mathrm{e}^{\operatorname{Logit}(P)}\right)$.

Receiver operating characteristic curve analysis was used to evaluate the accuracy of the risk prediction model. The area under the curve was 0.849 (95\% CI 0.772-0.926, $p<0.0001$, Figure 3). The cutoff value was 0.2825 , with a sensitivity of $91.4 \%$, a specificity of $65.1 \%$, a negative predictive value of $93.2 \%$, and a positive predictive value of $59.3 \%$.

\section{Validation of Model Stability in $\mathbf{3 2}$ Additional Patients}

To verify the stability of the risk prediction model, 32 patients older than 85 years from the First Medical Centre of Chinese People's Liberation Army General Hospital between January 
TABLE 3 | Univariate analysis of risk factors of linezolid-induced lactic acidosis.

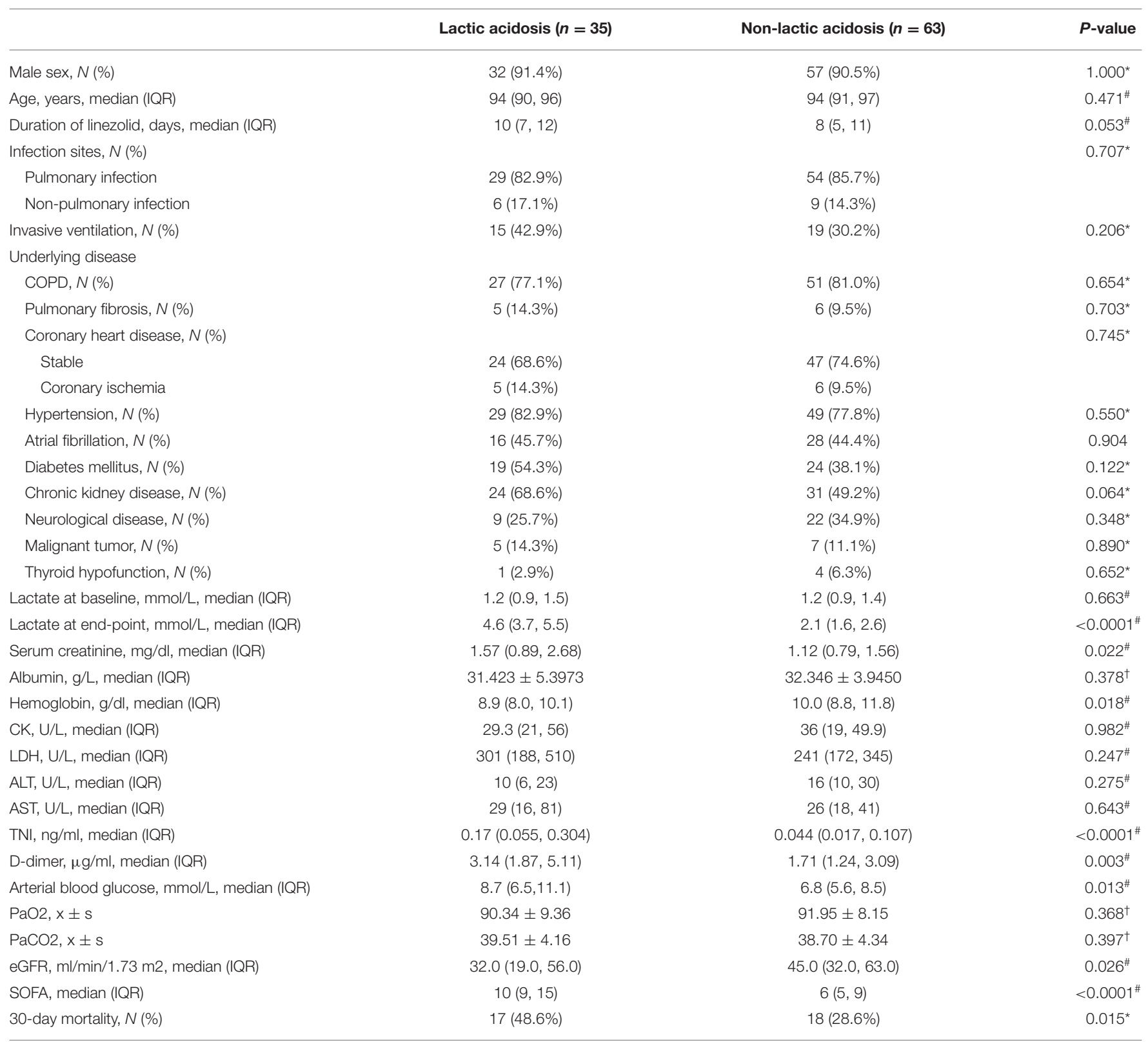

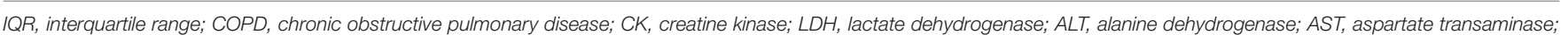
TNI, troponin I; pro-BNP, pro-brain natriuretic peptide; eGFR, estimate glomerular filtration rate; SOFA, sequential organ failure assessment; PaO 2 partial pressure of oxygen. ${ }^{*}$ Chi-square test.

"Mann-Whitney U test.

†t-test.

and October 2019 were included. All the patients had blood gas analysis and arterial lactate levels monitored during the use of linezolid. The gold standard for the diagnosis of LILA was the combination of blood gas analysis and arterial lactate measurements.

According to the four-fold table (Table 5), the sensitivity of the risk prediction model was $100 \%$, the specificity was $80 \%$, the negative predictive value was $100 \%$, and the positive predictive value was $58.3 \%$.

\section{DISCUSSION}

Linezolid has been used as a powerful medicine to treat multidrug-resistant gram-positive bacterial infections; however, this type of antibiotic could cause type B lactic acidosis due to inhibition of mitochondrial oxidative phosphorylation in the absence of apparent tissue hypoxia. LILA in the elderly has not been deeply concerned for a long time until a notable incidence of LILA was reported. 


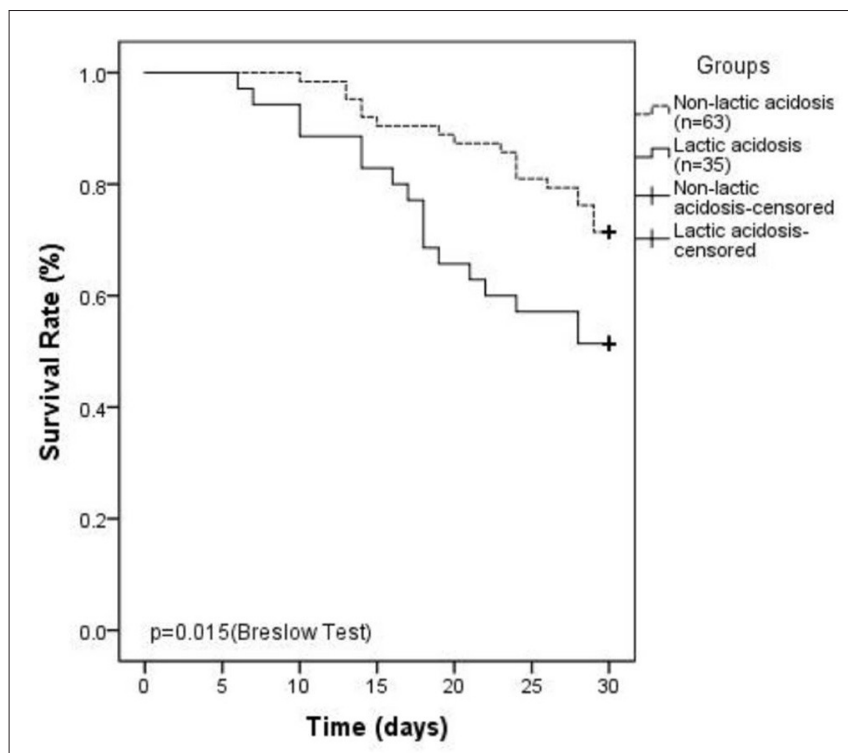

FIGURE 2 | Kaplan-Meier plot showing 30-day survival ratesinlinezolid-induced lactic acidosis group is lower than that in non-lactic acidosis group (48.6 vs. $28.6 \%, P=0.015$ ).

TABLE 4 | Multivariate analyses of risk factors for linezolid-induced lactic acidosis.

\begin{tabular}{lcc}
\hline & OR $\mathbf{( 9 5 \% ~ C l )}$ & $P$-value \\
\hline Duration of linezolid, $\geq 9$ days & $3.541(1.161-10.793)$ & 0.026 \\
Arterial blood glucose, $\geq 8 \mathrm{mmol} / \mathrm{L}$ & $4.548(1.507-13.725)$ & 0.007 \\
SOFA & $1.429(1.213-1.685)$ & $<0.0001$
\end{tabular}

LILA was supposed to affect the survival of patients in clinical practice.

In the current study, $35.7 \%$ of very elderly patients (older than 85 years) receiving linezolid therapy were observed with lactic acidosis. This overall percentage of LILA was slightly higher than previously reported, $6.8 \%$ in Im's study and $10.6 \%$ in Mori's study in all age groups (17-20). The reason for the higher percentage of LILA could be partly caused by the patients with mild disease who were excluded from the study due to the absence of blood gas monitoring. The very elderly population included in our study had relatively severe disease and multiple diseases or called comorbidities. In previous studies, those patients whose lactate levels were not being monitored were included in the non-lactic acidosis group, which might also lead to the underestimation of LILA incidence (20).

In our research, we found that the 30-day mortality rate was $48.6 \%$ in the lactic acidosis group, which was significantly higher than that in the control group $(p<0.05)$. In a systematic review and meta-analysis of 47 cases of LILA retrieved from PubMed, $25.5 \%$ of the patients died, indicating a high risk of mortality associated with LILA (21).

Previous reports have shown that LILA is associated with a longer duration of medicine therapy $(15,16,22-24)$. In a

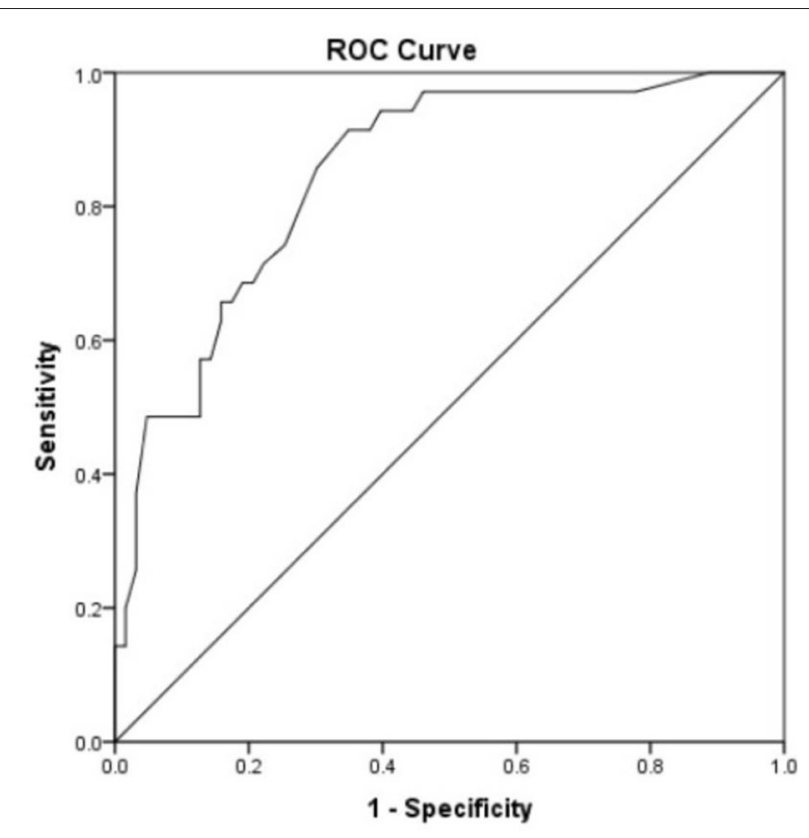

FIGURE 3 | Receiver operating characteristic curve analysis was used to evaluate the accuracy of the risk prediction model. Area under the curve was 0.849 with $95 \% \mathrm{Cl}(0.772-0.926)(p<0.0001)$. Cutoff was 0.2825 with a sensitivity of $91.4 \%$, a specificity of $65.1 \%$, a negative predictive value of $93.2 \%$, and a positive predictive value of $59.3 \%$.

retrospective study, duration of linezolid therapy $>6$ weeks was a risk factor for LILA (19), but LILA has been reported to occur after a shorter duration of linezolid therapy ( $4 \mathrm{~h}-7$ days) $(21,25-28)$ and even earlier in children with a median time of $2(1,13)$ days $(29)$. Del Pozo had shown that the median duration of the administration of linezolid in patients with lactic acidosis was 8 days (20). We found that the duration of linezolid therapy $\geq 9$ days was a risk factor for LILA in the very old population, suggesting that LILA in the very elderly population could occur after a short course of medication, although the underlying mechanism has not yet been clarified. Therefore, early and routine monitoring of lactate levels and blood gases is necessary for geriatric wards.

We also discovered that when the arterial blood glucose level was $\geq 8 \mathrm{mmol} / \mathrm{L}$, the risk of lactic acidosis was elevated. In the absence of oxygen or mitochondrial oxygen use disorders, glucose produces lactic acid through anaerobic fermentation glycolysis. This could be the major cause of why lactic acidosis is prone to occur with elevated blood glucose.

A high SOFA score could also be a risk factor for LILA, and the risk of lactic acidosis increased 0.429 times for every one-point increase in the SOFA score, suggesting that very elderly patients with high SOFA scores are more prone to lactic acidosis. One study of 10 cases of LILA found that a SOFA score $\geq 11$ and the duration of linezolid therapy $\geq 7$ days were not risk factors for LILA (20). This was inconsistent with our results, which may be related to the univariate analysis used in previous studies. 
TABLE 5 | Thirty-two patients older than 85 years verified the stability of the risk prediction model.

\begin{tabular}{|c|c|c|c|}
\hline \multirow[t]{2}{*}{ Risk prediction mode } & \multicolumn{2}{|c|}{ Gold standard } & \multirow[t]{2}{*}{ Total } \\
\hline & Lactate acidosis & Non-lactate acidosis & \\
\hline Lactate acidosis & 7 & 5 & 12 \\
\hline Non-lactate acidosis & 0 & 20 & 20 \\
\hline Total & 7 & 25 & 32 \\
\hline
\end{tabular}

Renal dysfunction might affect the excretion of linezolid, leading to the increased blood concentration of linezolid, and thereby induced LILA. Del Pozo (20) found that an eGFR $\leq 30$ $\mathrm{ml} / \mathrm{min}(\mathrm{OR}, 7.4 ; 95 \% \mathrm{CI}, 1.0-84.4, p=0.02)$ was a risk factor for LILA; however, we did not find that the eGFR was associated with LILA. Actually, the non-renal clearance rate of linezolid was $65 \%$. Furthermore, $30 \%$ of lactic acid clearance occurs in the kidney. Unless the lactic acid level is above $6-10 \mathrm{mmol} / \mathrm{L}$, it will not start to be excreted by the kidneys (1). Therefore, eGFR may only be associated with severe hyperlacticacidemia. In our study, lactate levels were mostly mildly to moderately elevated; no correlation was confirmed between LILA and eGFR.

LILA may be associated with linezolid overexposure. In a case report, patients who received regular doses of linezolid $(600 \mathrm{mg}$ twice daily) with significant plasma overexposure to linezolid (minimum concentration, $26.99 \mathrm{mg} / \mathrm{L}$ ) developed significant lactic acidosis (26). A recent study showed that patients $\geq 80$ years had concentrations three times higher compared with patients $<40$ years, suggesting a positive correlation between linezolid concentrations and patient age (30). This may be why the high incidence and early onset of LILA in elderly patients in our study. More data are needed to illustrate the correlation between the concentration of linezolid and LILA.

Based on the multivariate logistic regression analyses, we established a risk prediction model for the occurrence of LILA with high sensitivity and specificity (91.4 and 65.1\%, respectively), with a cutoff value of 0.2825 . Verification of the model in 32 patients from another medical center showed that it was very stable. Therefore, the risk prediction model can be applied to the very elderly population.

The mechanism underlying LILA is still unclear. Human cells only contain L-lactate dehydrogenase that exclusively synthesizes L-lactic acid. Some colonic carbohydrate-fermenting bacteria produce $\mathrm{D}$-lactic acid by $\mathrm{D}$-lactate dehydrogenase. The usual lactic acid laboratory tests cannot detect D-lactic acid. Therefore, linezolid might induce the production of Llactic acid by human cells. Linezolid inhibits $23 \mathrm{~S}$ ribosomal RNA (rRNA) from the 50S subunit of the bacterial ribosome, similar to human mitochondrial 16S rRNA. Hence, linezolid might produce toxic mitochondrial effects by binding to human mitochondrial 16S rRNA and inhibiting mitochondrial protein synthesis (24). Human mitochondrial DNA polymorphisms (A2706G and G3010A) have been found associated with LILA $(31,32)$, although this finding remains controversial due to the high frequency (up to $80 \%$ ) of the polymorphisms and the relatively rare occurrence of LILA (33).
To the best of our knowledge, this is the first study to analyze the occurrence of LILA in a large sample of patients older than 85 years. Moreover, we established a risk prediction model to predict the occurrence of LILA. There are some limitations to this study. First, as a retrospective study, the concentration of linezolid was not monitored. A recent preliminary result by our group showed that the trough concentrations of linezolid in elderly patients were more than $8 \mathrm{mg} / \mathrm{L}$. Further studies are of great interest soon. Second, the included patients of very old age presenting relatively severe illness might contribute to the mortality. Third, the sample size was relatively small, and other adverse effects of linezolid such as thrombocytopenia, erythrocytopenia, and nervous lesion were worth further investigation. Furthermore, the mechanism underlying LILA needs to be further studied.

\section{CONCLUSION}

This study identified the risk factors for LILA and established a stable risk prediction model. LILA can occur in very elderly patients after a relatively shorter duration of linezolid, indicating that the close monitoring of blood gases and arterial lactate levels during the administration of linezolid is necessary.

\section{DATA AVAILABILITY STATEMENT}

The original contributions presented in the study are included in the article/Supplementary Material, further inquiries can be directed to the corresponding author/s.

\section{ETHICS STATEMENT}

The studies involving human participants were reviewed and approved by the ethics committee of Chinese People's Liberation Army (PLA) General Hospital (Ethical approval number: S2018-162-01). Written informed consent for participation was not required for this study in accordance with the national legislation and the institutional requirements.

\section{AUTHOR CONTRIBUTIONS}

HL, GX, and TL contributed to the study design. JWa and YQ contributed to the collection of clinical data. HL, XF, and ML 
contributed to the data analysis. TL, $\mathrm{CH}$, and JWu drafted the article. All authors contributed to the article and approved the submitted version.

\section{ACKNOWLEDGMENTS}

This manuscript has been released as a preprint at Research Square (34). The authors are grateful to all participants, laboratory staff, and professionals involved

\section{REFERENCES}

1. Seheult J, Fitzpatrick G, Boran G. Lactic acidosis: an update. Clin Chem Lab Med. (2017) 55:322-3. doi: 10.1515/cclm-2016-0438

2. Vincent JL, Quintairos E Silva A, Couto L Jr, Taccone FS. The value of blood lactate kinetics in critically ill patients: a systematic review. Crit Care. (2016) 20:257. doi: 10.1186/s13054-016-1403-5

3. Jansen TC, van Bommel J, Schoonderbeek FJ, Sleeswijk Visser SJ, van der Klooster JM, Lima AP, et al. LACTATE study group. Early lactateguided therapy in intensive care unit patients: a multicenter, open-label, randomized controlled trial. Am J Respir CritCare Med. (2010) 182:75261. doi: 10.1164/rccm.200912-1918OC

4. Nichol AD, Egi M, Pettila V, Bellomo R, French C, Hart G, et al. Relativehyperlactatemia and hospital mortality in critically ill patients: aretrospective multi-centre study. Crit Care. (2010) 14:R25. doi: 10.1186/cc8888

5. Singer M, Deutschman CS, Seymour CW, Shankar-Hari M, Annane D, Bauer $\mathrm{M}$, et al. The third international consensus definitions for sepsis and septic shock (sepsis-3). JAMA. (2016) 315:801-10. doi: 10.1001/jama. 2016.0287

6. Brickner SJ, Barbachyn MR, Hutchinson DK, Manninen PR. Linezolid (ZYVOX), the first member ofa completely new class of antibacterial agents for treatment of serious gram-positive infections. J Med Chem. (2008) 51:1981-90. doi: 10.1021/jm800038g

7. Ahedi Bialvaei A, Rahbar M, Yousefi M, Asgharzadeh M, Samadi Kafil H. Linezolid: a promising option in the treatment of Grampositives. J Antimicrob Chemother. (2017) 72:354-64. doi: 10.1093/jac/ dkw450

8. Tang S, Yao L, Hao X, Zhang X, Liu G, Liu X, et al. Efficacy, safety and tolerability of linezolid for the treatment of XDR-TB: a study in China. Eur Respir J. (2015) 45:161-70. doi: 10.1183/09031936.00035114

9. Vinh DC, Rubinstein E. Linezolid: a review of safety and tolerability. J Infect. (2009) 59:S59-74. doi: 10.1016/S0163-4453(09) 60009-8

10. Kreps EO, Brown L, Rennie IG. Clinical recovery in linezolid-induced optic nerve toxicity. Acta Ophthalmol. (2017) 95:e341-2. doi: 10.1111/aos. 13237

11. Narita M, Tsuji BT, Yu VL. Linezolid-associated peripheral and optic neuropathy, lactic acidosis, and serotonin syndrome. Pharmacotherapy. (2007) 27:1189-97. doi: 10.1592/phco.27. 8.1189

12. Bobylev I, Maru H, Joshi AR, Lehmann HC. Toxicity to sensory neurons and Schwann cells in experimental linezolid-induced peripheral neuropathy. J Antimicrob Chemother. (2016) 71:685-91. doi: 10.1093/jac/ dkv386

13. Bressler AM, Zimmer SM, Gilmore JL, Somani J. Peripheral neuropathy associated with prolonged use of linezolid. Lancet Infect Dis. (2004) 4:52831. doi: 10.1016/S1473-3099(04)01109-0

14. Ferry $\mathrm{T}$, Ponceau $\mathrm{B}$, Simon $\mathrm{M}$, Issartel $\mathrm{B}$, Petiot $\mathrm{P}$, Boibieux A, et al. Possibly linezolid-induced peripheral and central neurotoxicity: report of four cases. Infection. (2005) 33:151-4. doi: 10.1007/s15010-0054057-9

15. Apodaca AA, Rakita RM. Linezolid-induced lactic acidosis. $N$ Engl J Med. (2003) 348:86-7. doi: 10.1056/NEJM200301023480123 in the study for their valuable contributions. We thank American Journal Experts for editing the English draft of this article.

\section{SUPPLEMENTARY MATERIAL}

The Supplementary Material for this article can be found online at: https://www.frontiersin.org/articles/10.3389/fmed. 2021.604680/full\#supplementary-material

16. Boutoille D, Grossi O, Depatureaux A, Tattevin P. Fatal lactic acidosis after prolonged linezolid exposure for treatment of multidrug-resistant tuberculosis. Eur J Intern Med. (2009) 20:e134-5. doi: 10.1016/j.ejim.2008.12.002

17. Beekmann SE, Gilbert DN, Polgreen PM. Toxicity of extended courses of linezolid: results of an Infectious Diseases Society of America Emerging Infections Network survey. Diagn Microbiol Infect Dis. (2008) 62:40710. doi: 10.1016/j.diagmicrobio.2008.08.009

18. Garrabou G, Soriano A, López S, Guallar JP, Giralt M, Villarroya F, et al. Reversible inhibition of mitochondrial protein synthesis during linezolidrelated hyperlactatemia. Antimicrob Agents Chemother. (2007) 51:9627. doi: 10.1128/AAC.01190-06

19. Im JH, Baek JH, Kwon HY, Lee JS. Incidence and risk factors of linezolid-induced lactic acidosis. Int $J$ Infect Dis. (2015) 31:47-52. doi: 10.1016/j.ijid.2014.12.009

20. Mori N, Kamimura Y, Kimura Y, Hirose S, Aoki Y, Bito S. Comparative analysis of lactic acidosis induced by linezolid and vancomycin therapy using cohort and case-control studies of incidenceand associated risk factors. Eur J Clin Pharmacol. (2018) 74:405-11. doi: 10.1007/s00228-017-2377-1

21. Mao Y, Dai D, Jin H, Wang Y. The risk factors of linezolidinduced lactic acidosis: a case report and review. Medicine. (2018) 97:e12114. doi: 10.1097/MD.0000000000012114

22. De Vriese AS, Coster RV, Smet J, Seneca S, Lovering A, Van Haute LL, et al. Linezolid-induced inhibition of mitochondrial protein synthesis. Clin Infect Dis. (2006) 42:1111-7. doi: 10.1086/501356

23. Sawyer AJ, Haley HL, Baty SR, McGuffey GE, Eiland EH. Linezolid-induced lactic acidosis corrected with sustained low-efficiencydialysis: a case report. Am J Kidney Dis. (2014) 64:457-9. doi: 10.1053/j.ajkd.2014.04.032

24. Velez JC, Janech MG. A case of lactic acidosis induced by linezolid. Nat Rev Nephrol. (2010) 6:236-42. doi: 10.1038/nrneph. 2010.20

25. Contou D, Fichet J, Grimaldi D, Cariou A. Early life-threatening lactic acidosis following a single infusion of linezolid. Int $J$ Antimicrob Agents. (2011) 38:84-5. doi: 10.1016/j.ijantimicag.2011. 01.025

26. Pea F, Scudeller L, Lugano M, Baccarani U, Pavan F, Tavio M, et al. Hyperlactacidemia potentially due to linezolid overexposure in a livertransplant recipient. Clin Infect Dis. (2006) 42:434-5. doi: 10.1086/ 499533

27. Su E, Crowley K, Carcillo JA, Michaels MG. Linezolid and lactic acidosis: a role for lactate monitoring with long-termlinezolid use in children. Pediatr Infect Dis J. (2011) 30:804-6. doi: 10.1097/INF.0b013e3182186035

28. Protti A, Ronchi D, Bassi G, Fortunato F, Bordoni A, Rizzuti T, et al. Changes in whole-body oxygen consumption and skeletal musclemitochondria during linezolid-induced lactic acidosis. Crit Care Med. (2016) 44:e57982. doi: 10.1097/CCM.0000000000001478

29. Ozkaya-Parlakay A, Kara A, Celik M, Ozsurekci Y, Karadag Oncel E, Ceyhan M, et al. Early lactic cidosis associated with linezolid therapy in paediatricpatients. Int J Antimicrob Agents. (2014) 44:3346. doi: 10.1016/j.ijantimicag.2014.06.017

30. Cattaneo D, Gervasoni C, Cozzi V, Castoldi S, Baldelli S, Clementi E. Therapeutic drug management of linezolid: a missed opportunity for clinicians? Int J Antimicrob Agents. (2016) 48:728-31. doi: 10.1016/j.ijantimicag.2016.08.023 
31. Del Pozo JL, Fernández-Ros N, Sáez E, Herrero JI, Yuste JR, Banales JM. Linezolid-induced lactic acidosis in two liver transplant patients with the mitochondrial DNA A2706G polymorphism. Antimicrob Agents Chemother. (2014) 58:4227-9. doi: 10.1128/AAC.02856-14

32. Palenzuela L, Hahn NM, Nelson RP Jr, Arno JN, Schobert C, Bethel $\mathrm{R}$, et al. Does linezolid cause lactic acidosis by inhibiting mitochondrial proteinsynthesis? Clin Infect Dis. (2005) 40:e113-6. doi: 10.1086/430441

33. Santini A, Ronchi D, Garbellini M, Piga D, Protti A. Linezolid-induced lactic acidosis: the thin line between bacterial and mitochondrial ribosomes. Expert Opin Drug Saf. (2017) 16:833-43. doi: 10.1080/14740338.2017.1335305

34. Liu T, Li H, Liu M, Wang J, Fang X. The risk factors for linezolid-induced lactic acidosis in patients older than 85 years. Res Square [Preprint]. (2020).
Conflict of Interest: The authors declare that the research was conducted in the absence of any commercial or financial relationships that could be construed as a potential conflict of interest.

Copyright (c) $2021 \mathrm{Liu}, \mathrm{Hu}, \mathrm{Wu}$, Liu, Que, Wang, Fang, Xu and Li. This is an open-access article distributed under the terms of the Creative Commons Attribution License (CC BY). The use, distribution or reproduction in other forums is permitted, provided the original author(s) and the copyright owner(s) are credited and that the original publication in this journal is cited, in accordance with accepted academic practice. No use, distribution or reproduction is permitted which does not comply with these terms. 